\title{
Computational Upscaling of Inertia Effects from Porescale to Mesoscale
}

\author{
Małgorzata Peszyńska ${ }^{1}$, Anna Trykozko ${ }^{2}$, and Kyle Augustson ${ }^{3}$ \\ 1 Oregon State University, Department of Mathematics, \\ mpesz@math. oregonstate.edu \\ 2 Interdisciplinary Centre for Math. and Comp. Modeling, University of Warsaw \\ aniat@icm.edu.pl \\ 3 University of Colorado at Boulder \\ kyle.augustson@Colorado.edu
}

\begin{abstract}
We propose algorithms for computational upscaling of flow from porescale (microscale) to lab scale (mesoscale). In particular, we solve Navier-Stokes equations in complex pore geometries and average their solutions to derive properties of flow relevant at lab scale such as permeability and inertia coefficients. We discuss two variants of traditional discretizations: a simple algorithm which works well in periodic isotropic media and can be used when coarse approximations are needed, and a more complex one which is well suited for nonisotropic geometries. Convergence of solutions and averaging techniques are major concerns but these can be relaxed if only mesoscopic parameters are needed. The project is a proof-of-concept computational laboratory for porous media which delivers data needed for mesoscale simulations by performing microscale computational simulations.
\end{abstract}

\section{Introduction}

Computational modeling of flow in porous media such as aquifers and oil-gas reservoirs has been constrained until recently to the scales of physical observation and of experiments such as Darcy-scale (lab-scale $\equiv$ mesoscale). For simulations in large porous reservoirs it has been necessary to upscale the models and parameters of flow to macroscale.

In this paper we pursue the upscaling from microscale, i.e., porescale to mesoscale (lab or Darcy scale). While relevant mathematical theory was developed decades ago via homogenization [1] and volume-averaging [2, the computational modeling at porescale had remained unfeasible until recently when advances in micro-imaging were accompanied by increases in computational power and development of discrete models such as network and lattice models 3456].

In this paper we are interested in continuum models, i.e., traditional discretizations of partial differential equations adapted to porescale such as studies in 78 . We investigate conditions under which simple algorithms can be used efficiently to deliver reliable quantitative information from microscale to mesoscale. Our project can be seen as a first step of a computational laboratory for modeling

G. Allen et al. (Eds.): ICCS 2009, Part I, LNCS 5544, pp. 695-704, 2009.

(C) Springer-Verlag Berlin Heidelberg 2009 
flow over a range of scales; the model at mesoscale can be further upscaled to macroscale following e.g. 910.

The following problem is of interest for stationary incompressible viscous flow. It is well known that linear flow models are not valid beyond certain Reynolds numbers 11,12 13 14 15 16]. At porescale the relevant linear and nonlinear models are the Stokes and Navier-Stokes equations, respectively. At mesoscale those are Darcy and non-Darcy models where it is now believed that in the nonlinear laminar regime it is the inertia rather than micro-turbulence effects that are most important. However, the identification of a particular transition regime between Darcy and non-Darcy flow model as well as a universal mathematical form of an anisotropic non-Darcy model are still a subject of current research and controversies [21617/8192021], and the values of associated coefficients reported in literature vary significantly.

Consider then a scenario in which data for linear flow at Darcy scale, i.e. permeability $\mathbf{K}$, is known, but no data for modeling inertia effects (denoted by $\beta$ ) is available. We propose to i) use a computational porescale model with inertia effects from which we ii) derive data $\beta$ for nonlinear models at Darcy scale. In i)-ii) we focus on $2 \mathrm{D}$ porescale models with isotropic mesoscale but iii) one can extend i)-ii) to anisotropic nonlinear laws at mesoscale that emerge from complicated anisotropic porescale geometries, and thereby aid current theoretical developments.

The main difficulties of i)-iii) are the following. First, standard discretization techniques for Navier-Stokes equations are well studied but their use in complex geometries requires fine grids and is in general nontrivial. Second, calculating average quantities from computational data is only superficially straightforward since the stability of results with respect to grids and algorithms over a large range of Reynolds numbers must be ensured. Next, as concerns iii), realistic data on porescale geometries such as [5] should be used, and their uncertainty, and dependence of results on averaging regions needs to be accounted for. Finally, computational efficiency of the proposed "on-demand" porescale modeling laboratory must be considered.

In this paper we focus on a proof-of-concept realization of i)-ii); details on iii) will be addressed in a forthcoming paper. In Sections 2 and 3 we describe the relevant physical and computational models, respectively. In Section 4 we propose the method of upscaling and in Section 5 we discuss the results.

\section{Computational Models}

Let $\Omega \subset \mathbb{R}^{d}, d=2,3$, be an open bounded domain occupied by porous medium and the fluid within. Let $\Omega_{F} \subsetneq \Omega$ be the part of $\Omega$ occupied by the fluid, that is, the domain of flow, and let rock (solid) part be $\Omega_{R}=\Omega \backslash \Omega_{F}$. Let $\partial \Omega$ denote the boundary of $\Omega$, and let $\Gamma=\partial \Omega_{F} \backslash \partial \Omega$ be the interior boundary (between rock and fluid domains) while the external boundary of flow $\partial \Omega_{F} \cap \partial \Omega$ is divided into inflow $\Gamma_{\text {in }}$ and outflow $\Gamma_{\text {out }}$ parts. We also denote by $\eta$ the unit outward normal to the boundary and by $\tau$ the unit tangent. For simplicity no special notation is used for numerical solutions. 
Flow at porescale. We consider an incompressible Newtonian fluid of velocity $\mathbf{u}$ and pressure $p$ flowing in $\Omega_{F}$. The fluid's viscosity is denoted by $\mu$ and the density $\rho \equiv 1$. We consider flow driven primarily by external boundary conditions, such as in a lab core. We prescribe velocity at the inlet and impose an outflow condition at the outlet. On internal boundaries we assume no-slip condition $\mathbf{u}=0$. We assume that the Reynolds number $\mathbf{R e}$ is correlated to the magnitude of inflow velocities.

At microscale (porescale), for steady-state flow, in the absence of forces and mass source/sink terms, the momentum and mass conservation in Eulerian frame are expressed by Navier-Stokes equations and continuity equation [14.

$$
\begin{aligned}
\mathbf{u} \cdot \nabla \mathbf{u}-\mu \triangle \mathbf{u} & =-\nabla p \\
\nabla \cdot \mathbf{u} & =0
\end{aligned}
$$

In 2D $(d=2)$ it is convenient to consider the formulation in terms of the vorticity vector $\omega=\nabla \times \mathbf{u}$ and the (scalar) stream function $\psi$ defined by $\mathbf{u}=\nabla \times \psi[14$. Taking $\nabla \times$ equation (1) and noticing $\nabla \times(\nabla p)=0$, one obtains the system

$$
\begin{aligned}
\boldsymbol{u} \cdot \nabla \boldsymbol{\omega} & =\mu \Delta \boldsymbol{\omega}, \\
\Delta \psi & =-\omega .
\end{aligned}
$$

The last equation follows from standard calculation $\boldsymbol{\omega}=\nabla \times(\nabla \times \boldsymbol{\psi})=\nabla(\nabla$. $\boldsymbol{\psi})-\Delta \boldsymbol{\psi}$, which, with (2), for 2D flow reduces to (4). We can get $p$ from

$$
-\Delta p=(\nabla(\boldsymbol{u} \cdot \nabla)) \cdot \boldsymbol{u}=2\left(\frac{\partial u_{x}}{\partial x} \frac{\partial u_{y}}{\partial y}-\frac{\partial u_{x}}{\partial y} \frac{\partial u_{y}}{\partial x}\right)
$$

For small $\mathbf{R e}$ the nonlinear convective terms associated with $\mathbf{u} \cdot$ are dropped from (11) and (3) and we have the (linear) Stokes approximation

$$
-\mu \triangle \mathbf{u}=-\nabla p
$$

which is valid when viscous effects dominate in the flow. For larger $\mathbf{R e}$ the inertia effects associated with $\mathbf{u} \cdot$ cannot be neglected. We recall that, up to the definition/units of characteristic quantities, the linear laminar flow regime is for $\mathbf{R e}<1$, the nonlinear regime is for $1 \leq \mathbf{R e}<100$, and that turbulence may occur for $\mathbf{R e} \geq 100$ [1316]; however, turbulence rarely occurs in porous media.

Flow at Darcy scale. At mesoscale the boundaries between $\Omega_{F}$ and $\Omega_{R}$ are no more recognized, One considers an average pressure $P:=<p>$ and velocity (flux) $\mathbf{U}:=<\mathbf{u}>$ where the averages over a volume $V(\mathbf{x})$ centered at $\mathbf{x} \in \Omega$ are defined as $<q>_{V} \equiv<q>(x) \equiv \frac{1}{|V(x)|} \int_{V(x)} q(y) d y$. (In what follows we drop subscript $V$ ). Conservation of mass after averaging yields $\nabla \cdot\langle\mathbf{u}\rangle=0$; note that derivatives in $\nabla$ are taken with respect to large scale variable $\mathbf{x}$. The flow in $\Omega$ at mesoscale is driven by boundary conditions which are averages of porescale external boundary conditions. 
Darcy's law is a linear momentum equation at mesoscale which can be proven 221] to be an average of Stokes flow (6)

$$
\mu \mathbf{K}^{-1}<\mathbf{u}>=-\nabla<p>
$$

where the values of a symmetric permeability tensor $\mathbf{K}$ are measured in a lab and have been obtained experimentally for many porous materials [16]. K reflects geometry at porescale, and $\mathbf{K}^{-1}$ measures resistance of porous medium to the flow. For heterogenous media $\mathbf{K}=\mathbf{K}(\mathbf{x})$, for isotropic media $\mathbf{K}=K \mathbf{I}$. Due to large viscous dissipation and interstitial effects common in porous media, Darcy's law is a good approximation for a large class of flow phenomena.

In the nonlinear laminar regime with significant inertia, averaging (1) yields

$$
\mathcal{K}^{-1}(<\mathbf{u}>)<\mathbf{u}>=-\nabla<p>\text {. }
$$

The 1D model for $\mathcal{K}$ first proposed by Forchheimer [11] was $\mathcal{K}^{-1}(U):=\mu K^{-1}+$ $\beta|U|$, while multidimensional isotropic version [12161315] reads

$$
\mathcal{K}^{-1}(<\mathbf{u}>)<\mathbf{u}>:=\left(\mathbf{K}^{-1} \mu+\beta|<\mathbf{u}>|\right)<\mathbf{u}>=-<\nabla p>.
$$

The form of nonlinear map $\mathcal{K}$ for general anisotropic $2 \mathrm{D}$ and $3 \mathrm{D}$ media has been the subject of theoretical research for general anisotropic $2 \mathrm{D}$ and $3 \mathrm{D}$ media 17/821 18 2019. Even if the model (9) for $\mathcal{K}$ is accepted, the data for coefficient $\beta$ are not universally available and/or consistent.

Mathematical upscaling from micro- to mesoscale. There are essentially two methodologies that apply. The first, with the use of homogenization theory $(\mathrm{H})$ 221 , requires periodic geometry but gives elegant theorems on convergence of the averages of microscale quantities to the appropriate mesoscale quantities when the size of periodic cell goes to 0 . The second, volume averaging (VA), does not restrict geometry and proposes that the averaged quantities are reasonably stable if the averaging region ( $\mathrm{REV} \equiv$ Representative Elementary Volume) is large enough [19. However, it may be difficult to quantify what size of REV is sufficient; see [2324] for related work on elasticity and our forthcoming work.

\section{Computational Models for Porescale}

For the flow in $\Omega_{F}$ we consider two algorithms $\mathcal{H}$ and $\mathcal{V} \mathcal{A}$ described below which are useful in similar contexts as, respectively, the mathematical upscaling methods $\mathrm{H}$ and VA. For other algorithms see 2526 .

We illustrate the algorithms with the following scenario. All flow in $\Omega$ is from left to right. The pore geometries are idealized: we envision rock grains as very long cylinders so that every cross-section can be approximated by a $2 \mathrm{D}$ computational region with $\Omega_{R}$ being a union of solid disks replicated periodically. The ratio of disk diameters to the size of the period denoted by $D$ ranges from 0.6 to 0.9 in this study; see Fig. 1 and 3 . 
Algorithm $\mathcal{H}$. This simple algorithm solves for $(\omega, \psi)$ in $d=2$ and uses simple structured grids over $\Omega_{F}$ and therefore can be easily adapted to interpret data from porescale imaging [5] without significant investment of time in grid generation. It is based on a central finite difference formulation enhanced by treatment of boundary conditions and post-processing, following [27,28,29].

The discretization of (3) and (4) yields

$$
\begin{array}{r}
\left(\mu \Delta_{h}-\left(\boldsymbol{u} \cdot \nabla_{h}\right)\right) \omega=0, \\
\Delta_{h} \psi=-\omega .
\end{array}
$$

where the numerical Laplacian $\Delta_{h}$ has the usual 5-point stencil and the advective term is computed using second order central differences. The coupling in the model is resolved by iteration: given $\omega^{n}$, i) compute $\psi^{n+1}$ from (11), then ii) calculate velocity $\boldsymbol{u}^{n+1}$ from $\psi^{n+1}$, then iii) solve (10) for $\omega^{n+\frac{1}{2}}$, and finally iv) compute $\omega^{n+1}=\lambda \omega^{n+\frac{1}{2}}+(1-\lambda) \omega^{n}$ where $\lambda$ is the relaxation parameter.

In this algorithm steps i) and ii) require that boundary values of $\psi$ and $\omega$ are known, respectively. This is the most delicate part of the algorithm and crucial for porescale computations: an idea how to implement inlet, outlet, and no-slip conditions follows; we refer to [26 2829] for details.

For structured rectangular grids over a periodic cell $\Omega$, the boundary $\Gamma$ is composed of vertical and horizontal segments only, and the external boundary has either vertical or horizontal $\Gamma_{i n}, \Gamma_{\text {out }}$. Consider vertical $\Gamma_{i n}$. On inlet boundary, $\mathbf{u}=\left(u_{x}, 0\right)$ is given and hence $\psi$ has to be constant and given as the integral of $u_{x}$ while vorticity is given from (44). On the vertical portion of the outlet boundary we have $\frac{\partial \omega}{\partial x}=0$ and $\frac{\partial \psi}{\partial x}=0$.

In the interior, we have no-slip boundary conditions $\mathbf{u}=0$; it follows that $\psi \equiv$ const. To find useful conditions for $\omega$, we approximate its second derivative as follows. Consider Taylor expansion $\psi(x+\delta x, y)=\psi(x, y)+\delta x \frac{\partial \psi}{\partial x}+\frac{\delta x^{2}}{2} \frac{\partial^{2} \psi}{\partial x^{2}}+$ $O\left(\delta x^{3}\right)$. But $u_{y}=-\frac{\partial \psi}{\partial x}=0$ thus $-\omega=\frac{\partial^{2} \psi}{\partial x^{2}}=\frac{2(\psi(x+\Delta x, y)-\psi(x, y))}{\delta x^{2}}+O(\delta x)$.
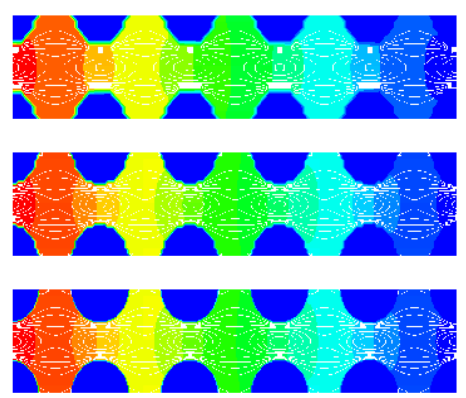
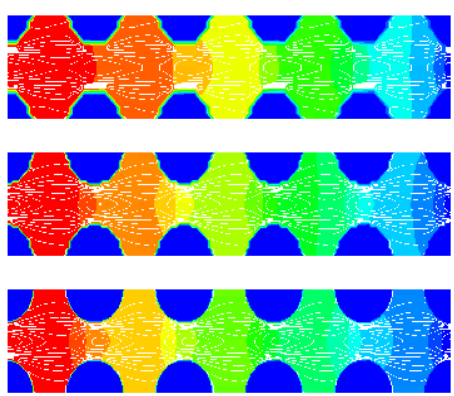

Fig. 1. Results of algorithm $\mathcal{H}, D=0.6$. Shown are profiles of $p$ overlaying contours of $\mathbf{u}$ for $\mathbf{R e}=1,100$, (left and right) on three grids: coarse, fine, and very fine (top to bottom). Even though the pointwise values appear unresolved on the coarse meshes, the computed averages and $\mathbf{K}$ and $\beta$ are stable on all grids. 

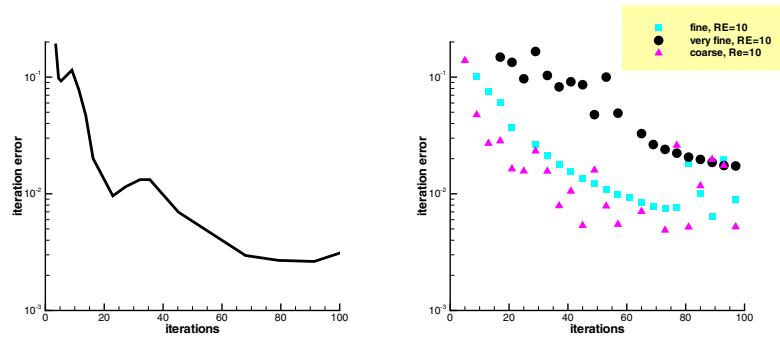

Fig. 2. Convergence of relaxation iteration in algorithm $\mathcal{H}$. Left: standard benchmark problem of Poiseille flow [26]. Right: flow in geometry as in Fig. 11 The iteration error (both) is defined as discrete $l_{2}$ norm of the stream function $\psi$.
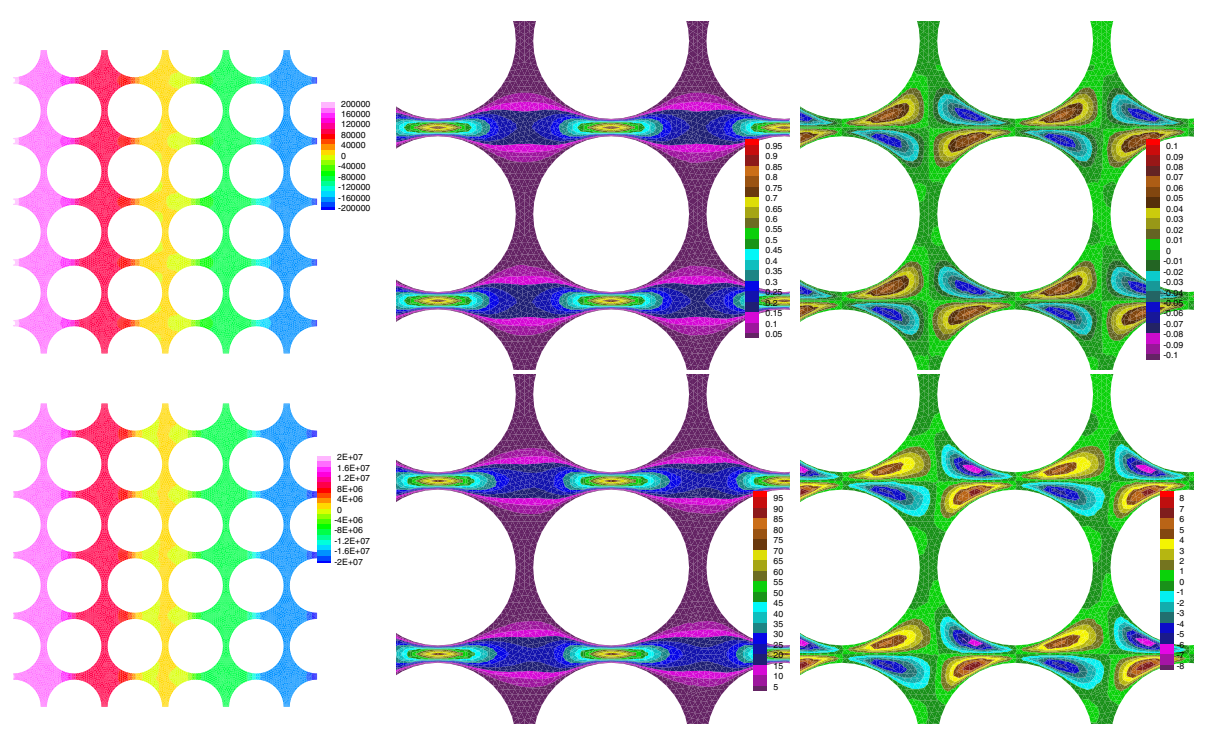

Fig. 3. Results of $\mathcal{V} \mathcal{A}$ for $D=0.9$ with $\mathbf{R e}=1$ (top) and $\mathbf{R e}=100$ (bottom). Shown are contours of pressure $p$ (left) and zoomed in velocity $\mathbf{u}$ profiles: component $x$ (middle) and $y$ (right).

To recover (post-process for) the velocities and pressures, we use central finite differences in the interior of the domain and appropriate one-sided differences at the boundary. The pressure is found from (5).

As seen in Fig. 1, the algorithm works reasonably well for a range of Reynolds numbers and grids that are not very fine. The difficulties arise since finding an optimal value of $\lambda$ in step iv) may be a problem; see Fig. 2. The simplicity of $\mathcal{H}$ is in that it consequently uses the same Poisson solver for which very efficient solvers and preconditioners 26/25] are available. As an alternative, a coupled solver for (10)-(11) can be written but this requires sophisticated nonsymmetric 
solvers and preconditioners. Overall, the algorithm $\mathcal{H}$ works well for small periodic domains $\Omega$ but may scale poorly to large regions, complex geometries, and large Re.

Algorithm $\mathcal{V} \mathcal{A}$. This algorithm solves for $(\mathbf{u}, p)$ and can be used in complicated geometries but requires substantial pre-processing; it follows an industry standard in computational fluid dynamics [30 31]; general unstructured grids can be used in 2D and 3D. We omit the details but provide an example which illustrates the grids and complexity of computations, see Fig. 3 .

\section{Upscaling Algorithms from Porescale to Mesoscale}

Strictly speaking, the work reported in this paper does not require any computations at mesoscale, i.e., in $\Omega$. However, keeping in mind our future goals, we choose to upscale from microscale to some chosen computational grid at mesoscale. In this paper we choose the conservative cell-centered finite difference method equivalent to lowest order Mixed Finite Element method on rectangles [32] this provides a bridge to macroscale following [910].

The idea is as follows: we impose a mesoscale cell-centered grid over $\Omega$ in a way which defines principal directions of flow that we anticipate will prevail at mesoscale (This may help to avoid handling full tensor $\mathbf{K}$ at mesoscale). With each center of mesocale grid $\left(X_{j}, Y_{j}\right)$, we have an associated cell $\Omega_{j}$ over which we average to get values $P_{j}$. Velocities are computed over unions of regions so that they are associated with locations "dual" to those for pressures [32]; see Fig. 4 for illustration. Ideally, the locations $\left(X_{j}, Y_{j}\right)$ coincide with centers of mass of $\Omega_{j}$ and the velocity components are computed in the direction of principal axis. However, there are ways to handle situations when this does not hold.

Assume now that porescale results $(\mathbf{u}, p)$ are available. We then compute $\mathbf{U}$ and $P$ as discussed above. Next, by inverse modeling, we identify resistance of the medium $\mathcal{K}^{-1}$ in the discrete counterpart of (8)
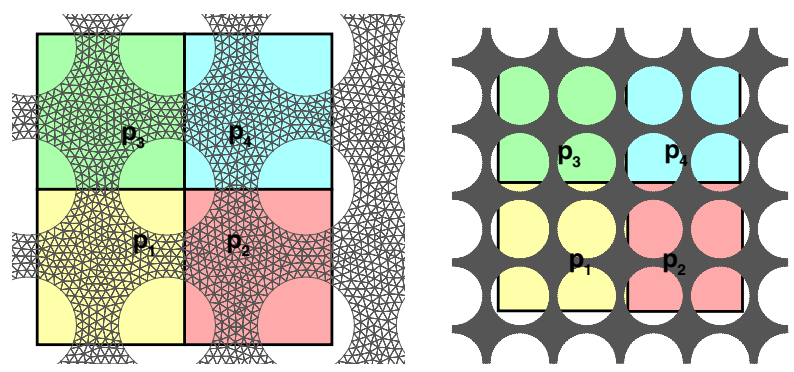

Fig. 4. Left: averaging region for a small periodic region $\Omega$, case $D=0.7$. The $x$ component $\mathbf{U}$ can be computed from averaging over regions $\Omega_{1}, \Omega_{3}$ and $\Omega_{2}, \Omega_{4}$. Right: general averaging region, case $D=0.9$. 

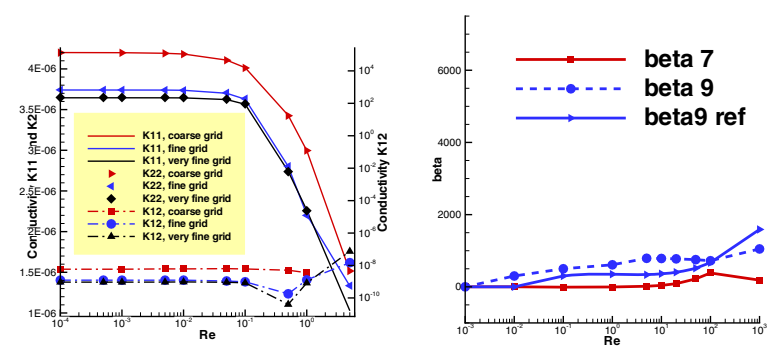

Fig. 5. Results of upscaling from porescale to lab scale. Left: dependence of $\mathbf{K}$ on the grid and $\mathbf{R e}$; notice isotropy at mesoscale revealed by equal diagonal and very small off-diagonal components of $\mathcal{K}$. Right: dependence of $\beta$ on the relative diameter $D \in(0.7,0.9)$ and on grid size (results computed for coarse and fine grids).

Note that for small Re, the resistance $\mathcal{K}^{-1}$ reduces to $\mathbf{K}^{-1}$ as in (7). Thus, if data is available for a large range of $\mathbf{R e}$ from creeping flow to nonlinear laminar regime, then one could hope to identify the appropriate model of tensor $\mathcal{K}$. In particular, if the medium is isotropic at mesoscale, then (9) is valid. In other words, given $\mathcal{K}$ and $\mathbf{K}$ and knowing $\mathbf{U}$, one can compute $\beta$ for any Re. Clearly if the model for $\mathcal{K}$ is valid and the computational algorithm is successful then $\beta$ remains reasonably constant throughout the nonlinear laminar regime; this appears true in our results, see Fig. 5 .

We stress that stability of $\mathbf{K}$ and $\beta$ is not guaranteed with just any adhoc averaging technique; in particular, the choice of REV, principal axis and their orientation, and of the boundary conditions, plays a significant role. In addition, there is currently no general explicit mathematical model and virtually no experimental work for anisotropic inertia represented by $\mathcal{K}$.

\section{Discussion}

Fluid flow in porespace is subject to viscous effects, inertia effects, and dissipation on the solid boundaries. In order to approximate the flow accurately for large Re, we need to ensure that the grid is fine enough in the channels where the solid boundaries are the closest to each other.

Using algorithm $\mathcal{H}$ we observed reasonable convergence for $D \leq 0.6$. However, more work needs to be done before the algorithm $\mathcal{H}$ can scale to more complicated geometries with $D>0.6$ and for large Re. In particular, we are considering a transient regularization of (3) which will help the convergence.

The use of algorithm $\mathcal{V} \mathcal{A}$ was promising for realistic porosities i.e. $D>0.9$. However, $\mathcal{V} \mathcal{A}$ requires care in gridding and monitoring convergence of the iterations. Here the difficulties are related to proper porescale grid definition with respect to principal axis. There is also the relative lack of availability of $\mathcal{V} \mathcal{A}$ due to its commercial implementation.

Overall, regardless of the porescale algorithm chosen, for some grids and some $\mathbf{R e}$, the profiles of $(\mathbf{u}, p)$ may reveal local instabilities. However, this does not 
necessarily lead to an instability of mesoscale properties at least with the averaging method that we proposed. In fact, $\mathbf{K}$ and $\beta$ appear stable for a large range of values of $\mathbf{R e}$ as well as appear convergent with respect to the grid size, see Fig. 5. We note that both $\mathbf{K}$ and $\beta$ are nonlinear quantities of interest in the sense of [24; see also [33] for recent related work on multiscale modeling.

Current and future work includes convergence analysis as well as serious computational studies aiding the theoretical modeling of tensor $\mathcal{K}$. Our project is a prototype of a computational laboratory which can provide on-demand model data for flow with inertia in porous media.

Acknowledgements. The work of Peszynska and Augustson was partially supported from NSF grant 0511190 and DOE grant 98089. The authors thank anonymous reviewers for additional references and comments that helped to improve the paper.

\section{References}

1. Tartar, L.: Incompressible fluid flow in a porous medium-convergence of the homogenization process. In: Nonhomogeneous media and vibration theory. Lecture Notes in Physics, vol. 127, pp. 368-377. Springer, Berlin (1980)

2. Ruth, D., Ma, H.: On the derivation of the Forchheimer equation by means of the averaging theorem. Transp. Porous Media 7(3), 255-264 (1992)

3. Schaap, M., Porter, M., Christensen, B.S.B., Wildenschild, D.: Comparison of pressure-saturation characteristics derived from computed tomography and Lattice-Boltzmann simulations. Water Resour. Res. 43 (2007)

4. Pan, C., Hilpert, M., Miller, C.: Pore-scale modeling of saturated permeabilities in random sphere packings. Physical Review E. 64 (2001)

5. Lindquist, W.B.: Network flow model studies and 3D pore structure. In: Fluid flow and transport in porous media: mathematical and numerical treatment. Contemp. Math, vol. 295, pp. 355-366. Amer. Math. Soc, Providence (2002)

6. Succi, S.: The Lattice Boltzmann equation for fluid dynamics and beyond. In: Numerical Mathematics and Scientific Computation. The Clarendon Press, Oxford University Press, New York (2001)

7. Andrade, J.S., Costa, U.M.S., Almeida, M.P., Makse, H.A., Stanley, H.E.: Inertial effects on fluid flow through disordered porous media. Phys. Rev. Lett. 82(26), $5249-5252(1999)$

8. Fourar, M., Lenormand, R., Karimi-Fard, M., Horne, R.: Inertia effects in high-rate flow through heterogeneous porous media. Transp. Porous Media 60(18), 353-370 (2005)

9. Durlofsky, L.J.: Numerical calculation of equivalent grid block permeability tensors for heterogeneous porous media. Water Resour. Res. 27(5), 699-708 (1991)

10. Garibotti, C., Peszynska, M.: Upscaling Non-Darcy Flow. Transport in Porous Media (published online March 13, 2009) doi:10.1007/s11242-009-9369-2

11. Forchheimer, P.: Wasserbewegung durch Boden. Zeit. Ver. Deut. Ing. (45), 17811788 (1901)

12. Ergun, S.: Fluid flow through packed columns. Chemical Engineering Progress 48, 89-94 (1952) 
13. Scheidegger, A.E.: The physics of flow through porous media. The Macmillan Co., New York (1960)

14. Batchelor, G.K.: An introduction to fluid dynamics. Cambridge (1999)

15. Dullien, F.: Porous media. Academic Press, San Diego (1979)

16. Bear, J.: Dynamics of Fluids in Porous Media. Dover, New York (1972)

17. Mei, C.C., Auriault, J.L.: The effect of weak inertia on flow through a porous medium. J. Fluid Mech. 222, 647-663 (1991)

18. Giorgi, T.: Derivation of Forchheimer law via matched asymptotic expansions. Transp. Porous Media 29, 191-206 (1997)

19. Bennethum, L.S., Giorgi, T.: Generalized Forchheimer equation for two-phase flow based on hybrid mixture theory. Transp. Porous Media 26(3), 261-275 (1997)

20. Chen, Z., Lyons, S.L., Qin, G.: Derivation of the Forchheimer law via homogenization. Transp. Porous Media 44(2), 325-335 (2001)

21. Huang, H., Ayoub, J.: Applicability of the Forchheimer equation for non-Darcy flow in porous media. SPE Journal (SPE 102715), 112-122 (2008)

22. Bensoussan, A., Lions, J.L., Papanicolaou, G.: Asymptotic analysis for periodic structures. North-Holland Publishing Co., Amsterdam (1978)

23. Moës, N., Oden, J.T., Vemaganti, K., Remacle, J.F.: Simplified methods and a posteriori error estimation for the homogenization of representative volume elements (RVE). Comput. Methods Appl. Mech. Engrg. 176(1-4), 265-278 (1999)

24. Oden, J.T., Vemaganti, K.S.: Estimation of local modeling error and goal-oriented adaptive modeling of heterogeneous materials. I. Error estimates and adaptive algorithms. J. Comput. Phys. 164(1), 22-47 (2000)

25. Wesseling, P.: Principles of computational fluid dynamics. Springer Series in Computational Mathematics, vol. 29. Springer, Berlin (2001)

26. Elman, H.C., Silvester, D.J., Wathen, A.J.: Finite elements and fast iterative solvers: with applications in incompressible fluid dynamics. Oxford University Press, New York (2005)

27. Landau, R.H., Páez, M.J.: Computational physics. Wiley, New York (1997)

28. Spotz, W.F., Carey, G.F.: High-order compact scheme for the steady streamfunction vorticity equations. Internat. J. Numer. Methods Engrg. 38(20), 3497$3512(1995)$

29. Weinan, E., Liu, J.G.: Vorticity boundary condition and related issues for finite difference schemes. J. Comput. Phys. 124(2), 368-382 (1996)

30. Patankar, S.V.: Numerical Heat Transfer and Fluid Flow FLUENT software, Hemisphere, Washington DC (1980)

31. Fluent Inc.: Fluent User's Guide, ver. 6.3 (2006)

32. Russell, T.F., Wheeler, M.F.: Finite element and finite difference methods for continuous flows in porous media. In: Ewing, R.E. (ed.) The Mathematics of Reservoir Simulation, pp. 35-106. SIAM, Philadelphia (1983)

33. Oden, J.T., Prudhomme, S., Romkes, A., Bauman, P.T.: Multiscale modeling of physical phenomena: adaptive control of models. SIAM J. Sci. Comput. 28(6), 2359-2389 (2006) 